\title{
(6) OPEN ACCESS \\ Minimal intervention delivered by 2-1-1 information and referral specialists promotes smoke-free homes among 2-1-1 callers: a Texas generalisation trial
}

\author{
Patricia Dolan Mullen, ${ }^{1}$ Lara S Savas, ${ }^{1}$ Łucja T Bundy, ${ }^{2}$ Regine Haardörfer, ${ }^{2}$ \\ Mel Hovell, ${ }^{3}$ Maria E Fernández, ${ }^{1}$ Jo Ann A Monroy, ${ }^{1}$ Rebecca S Williams, ${ }^{4}$ \\ Matthew W Kreuter, ${ }^{5}$ David Jobe, ${ }^{6}$ Michelle C Kegler ${ }^{2}$
}

${ }^{1}$ Center for Health Promotion and Prevention Research, University of Texas School of Public Health, Houston, Texas, USA

2Department of Behavioral Sciences and Health Education, Emory Prevention Research Center, Rollins School of Public Health, Emory University, Atlanta, Georgia, USA

${ }^{3}$ Center for Behavioral Epidemiology and Community Health, Graduate School of Public Health, San Diego State University, San Diego, California, USA

${ }^{4}$ Center for Health Promotion and Disease Prevention and Lineberger Cancer Center, University of North CarolinaChapel Hill, Chapel Hill, North Carolina, USA

${ }^{5}$ Health Communication Research Laboratory, Washington University, St. Louis, Missouri, USA ${ }^{6}$ 2-1-1 Texas/United Way HELPLINE, United Way of Greater Houston, Houston, Texas, USA

Correspondence to Dr Patricia Dolan Mullen, University of Texas School of Public Health, 7000 Fannin Street, Suite 2522, Houston, TX 77030, USA; Patricia.D. Mullen@uth.tmc.edu

Received 11 March 2016 Revised 1 August 2016 Accepted 2 August 2016

\section{(a) CrossMark}

To cite: Mullen PD, Savas LS, Bundy $Ł T$, et al. Tob Control 2016;25:110i18.

\section{ABSTRACT}

Background Replication of intervention research is reported infrequently, limiting what we know about external validity and generalisability. The Smoke Free Homes Program, a minimal intervention, increased home smoking bans by United Way 2-1-1 callers in randomised controlled trials in Atlanta, Georgia and North Carolina.

Objective Test the programme's generalisabilityexternal validity in a different context.

Methods A randomised controlled trial $(n=508)$ of English-speaking callers from smoking-discordant households ( $\geq 1$ smoker and $\geq 1$ non-smoker). 2-1-1 Texas/United Way HELPLINE call specialists serving the Texas Gulf Coast recruited callers and delivered three mailings and one coaching call, supported by an online tracking system. Data collectors, blind to study assignment, conducted telephone interviews 3 and 6 months postbaseline.

Results At 3 months, more intervention households reported a smoke-free home $(46.6 \%$ vs $25.4 \%$, $\mathrm{p}<0.0001$; growth model intent-to-treat $\mathrm{OR}=1.48,95 \%$ Cl 1.241 to $1.772, p<0.0001)$. At 6 months, selfreported full bans were $62.9 \%$ for intervention participants and $38.4 \%$ for controls $(O R=2.19)$. Texas trial participants were predominantly women $(83 \%)$, single-smoker households (76\%) and African-American $(65 \%)$; half had incomes $\leq$ US $\$ 10$ 000/year (50\%). Texas recruitment was $<50 \%$ of the other sites. Fewer callers reported having a smoker in the household. Almost twice the callers with a household smoker declined interest in the programme/study.

Conclusions Our findings in a region with lower smoking rates and more diverse callers, including English-speaking Latinos, support programme generalisability and convey evidence of external validity. Our recruitment experience indicates that site-specific adjustments might improve recruitment efficiency and reach.

Trial registration number NCT02097914, Results.

\section{INTRODUCTION}

Despite decreasing rates of secondhand smoke exposure (SHSe) among US non-smokers, 58 million adults and children were still exposed in 2011-2012, and households with young children, non-Latino African-Americans and adults with lower education levels and living in poverty had the highest risk for SHSe. ${ }^{1-6}$ Annually, SHSe causes 41000 deaths among non-smoking adults, 400 infant deaths and an estimated US\$5.6 billion loss in productivity. ${ }^{7}$

Household smoking bans decrease SHSe of nonsmokers, ${ }^{1}$ 9-11 decrease smokers' cigarette consumption and increase their quit attempts. ${ }^{10}$ 12-17 Yet, most intervention research to reduce SHSe has been conducted in clinical settings with relatively intensive counselling on exposure levels with parents of infants and of children who have asthma or are medically compromised. ${ }^{18-27}$

To increase protection of healthy children and non-smoking adults from SHSe, we developed the Smoke-Free Homes Program: Some Things are Better Outside. This is a minimally intensive programme with three mailings and a single counselling telephone call, implemented in partnership with 2-1-1 social services information and referral (I\&R) systems. Annually, US 2-1-1 I\&R specialists connect 15 million socioeconomically disadvantaged, uninsured/underinsured callers to community social and health resources, such as rent and utility assistance, healthcare referrals and case management. ${ }^{28}$ With its coverage of $93 \%$ of US communities, the 2-1-1 national I\&R system is a natural partner for interventions for vulnerable populations, including those with higher rates of tobacco use and smoking in the home. ${ }^{29-32}$

The programme's goal of home smoking bans was intended for smokers and non-smokers and was based on 90 interviews with low-income families in rural Georgia to understand household dynamics around creating smoking bans ${ }^{33}{ }^{34}$ and on a pilot study with 40 participants. ${ }^{35}$ As described in previous reports, ${ }^{32} 35$ the theoretical framework combined social cognitive theory and stages of change (transtheoretical model). Theory-based change methods ${ }^{36} 37$ included persuasion, role modelling, goal setting, environmental cues and written and oral reinforcement of actions taken to create a smoke-free home. ${ }^{38} 39$ Materials mailed at 2-week intervals focused on a five-step process for establishing a home smoking ban ${ }^{32} 35$ and included a five-step guide, a photo novella, family pledge, stickers, window cling signs and a fact sheet. A 15-20 min coaching call following the first mailing emphasised goal setting, problem solving and reinforcement and rehearsed barriers and possible solutions to creating a ban.

The Smoke-Free Homes research agenda included the pilot study, ${ }^{35}$ followed by three randomised trials. The vanguard trial conducted in 
Atlanta, Georgia, by Emory University researchers with United Way of Greater Atlanta 2-1-1 found a higher uptake of full bans 6 months postbaseline in the intervention group compared with the controls $(\mathrm{OR}=1.56){ }^{32}$ The second trial in North Carolina (NC) by the University of North Carolina-Chapel Hill researchers with the state United Way added elements of an effectiveness trial $^{40}$-delivery of the mailings and the coaching call by 2-1-1 I\&R specialists. The NC trial continued strong University support in training, certifying and supervising 2-1-1 staff. This trial relied on self-reported outcomes (table 1).

Atlanta had facilitated recruitment delivery of the mailings and follow-up data collection with a custom-built online tracking tool; the NC trial added the coaching call to the online tracking tool and 2-1-1 staff delivered the call. NC's results $(\mathrm{OR}=1.72)$ were similar to Atlanta's, after considering possible inflation for self-report and a more challenging population profile, for example, NC trial participants had more smokers in their household. ${ }^{43}$ To inform dissemination, our trial replicated the NC protocol in the Texas Gulf Coast 2-1-1 centre, which has a larger call volume $(25000$ calls/ day) and serves a more diverse population (more Latinos and Asians) and is set in an area with a lower smoking prevalence (Houston Standard Metropolitan Statistical Area $=15.6 \%$ and Corpus Christi Public Health Region=16.7\%). ${ }^{44}$

Intervention replication is reported infrequently, thus limiting what we know about the external validity and generalisability of the interventions. ${ }^{45}$ Failure to replicate initial studies in psychology and other disciplines and reported gaps between research and practice in public health ${ }^{46-50}$ have led to calls for greater emphasis on replication. Results from one modelling report suggest that at least three replications are needed to establish an effect, that is, internal validity. ${ }^{51}$ This analysis and other discussions coincide with calls to test generalisability (external validity) across varied settings, contexts, populations and times to establish a reliable evidence base for practice. ${ }^{474852}$

As implied above, the efficacy-effectiveness distinction is a continuum, not a dichotomy; ${ }^{4053}$ a test of generalisability should not change all variables at once, especially implementation fidelity. Thus, 'voltage drop', where efficacious programmes typically achieve a lower magnitude of effect in new settings and populations, is attributed to contextual issues, implementation, more heterogeneous participants and other factors that are sometimes, unfortunately, confounded in a single replication study. ${ }^{54}$

Trial replication across research teams, populations, locations and time involves systematically repeating the methods under varying contexts that involve systematic and random errors,

Table 1 Comparison of Atlanta and NC Trial elements and context

\begin{tabular}{|c|c|c|}
\hline Trial & Atlanta & North Carolina \\
\hline Recruiters & 2-1-1 staff & 2-1-1 staff \\
\hline Participants & $\begin{array}{l}\text { All eligible, consenting } \\
\text { callers }\end{array}$ & $\begin{array}{l}\text { All eligible, } \\
\text { consenting callers }\end{array}$ \\
\hline Intervention deliverers & University staff & 2-1-1 staff \\
\hline Follow-up interviewers & University staff & University staff \\
\hline Outcome measure & $\begin{array}{l}\text { Self-report+nicotine } \\
\text { monitors }\end{array}$ & Self-report \\
\hline $\begin{array}{l}2-1-1 \text { call volume (at the } \\
\text { time of the trial) }\end{array}$ & 1050/day & 341/day \\
\hline Area smoking rates & $\begin{array}{l}\text { Atlanta public health } \\
\text { region }=17.5 \%\end{array}$ & NC state $=21.2 \%{ }^{42}$ \\
\hline
\end{tabular}

which differ by site. These sources of errors can bias towards the null hypothesis by reducing intervention dose and fidelity (ie, effect size) or reducing study power to detect a similar effect. When three or more replications attain significant effects at all sites, the chance that all independent replications show significant findings is exceedingly low. ${ }^{51}$ It is this low probability of consistent findings in each different replication context that makes it possible to conclude external validity and to some degree construct (theoretical) validity.

Thus, the Texas trial was expected to serve as a robust test of recruitment generalisability, implementation fidelity and effectiveness by replicating the $\mathrm{NC}$ protocol and varying only the population and context. Continuity of the delivery system (2-1-1) supported by the protocol manual and online tracking system was expected to maintain implementation fidelity. In addition, this work provided the opportunity to compare intervention reach and delivery across studies and to suggest contextspecific adaptations.

\section{METHODS}

We conducted a single-blind, randomised controlled trial with 2-1-1 Texas/United Way HELPLINE Greater Houston (2-1-1 Texas) callers from March 2014 to November 2015. Eligible participants (one per household) were 18 years or older, could speak and understand English, were either a smoker living with one or more non-smokers (including children) or a non-smoker living with one or more smokers and resided in a home without a total smoking ban. Assessments were completed by 2-1-1 Texas staff at baseline and by University staff at 3 and 6 months postbaseline. All 2-1-1 Texas and University staff in the roles of recruiter, coach or follow-up interviewer completed five consecutive satisfactory recordings before certification; subsequently, $10 \%$ of calls were recorded and reviewed for protocol adherence.

All aspects of the study were managed via the custom-built, online tracking tool. The tool produced notifications when an outstanding task was due and when that activity was completed (or missed within the specified time), and it scheduled the next task. An address verification tool, SmartyStreets, ${ }^{55}$ embedded in the tracking tool checked for correct addresses at enrolment.

2-1-1 Texas is the largest US 2-1-1 centre by call volume (2500 calls/day). The United Way in Houston has operated a helpline since 1947, transitioning in 2002 to 2-1-1 and has become the area information centre for the Texas Gulf Coast, a 13-county region, which includes Houston and extends to Corpus Christi. 2-1-1 Texas employs 65 I\&R specialists. This trial continued an active partnership; ${ }^{29}{ }^{56-58}$ a subcontract compensated I\&R specialists' time to fulfil programme duties.

\section{Recruitment, enrolment and random assignment}

Atlanta and NC project coordinators conducted a 1-day, in-person training session to review and practice procedures and baseline interviews for I\&R specialists selected as recruiters $(n=5)$. After providing the standard service for non-crisis callers, recruiters described the study as promoting healthier homes by reducing SHSe and asked whether there was a smoker living in the caller's household. Those reporting one or more smokers were asked about their interest in participating in the programme/study and assessed for further eligibility and consent; the consent script mentioned US $\$ 25$ gift cards for baseline, and each completed the follow-up interview. The recruiter administered the baseline interview, and the tracking tool then randomly assigned participants to the control or intervention group. 


\section{Intervention delivery}

The content, number and timing of the three intervention mailings and single coaching call were the same as in the Atlanta ${ }^{32}$ and NC trials. ${ }^{43}$ In-person training by Atlanta and NC project coordinators prepared I\&R specialists selected as programme coaches ( $n=2$ females). Coaching calls were made 2 weeks after the first mailing, and 12 call attempts, 2 letters/text messages and, if available, 3 call attempts to an emergency contact were permitted. Following participant confirmation to begin, the coaching call typically took $10-20 \mathrm{~min}$ to complete.

\section{Follow-up data collection}

University staff who were blind to the study condition completed 20-30 min telephone interviews at 3 and 6 months postbaseline. The contact protocol included up to 12 call attempts, 2 mailed letters/text messages and up to 3 attempts to reach an alternant contact, triggered and monitored by the tracking tool.

\section{Measures}

Measures were specified in advance and based on standardised items, described by Kegler et $a l^{32}$ used in the previous trials and were collected at the same intervals and manner. The exception was that Atlanta had used home air nicotine monitors to validate 3 -month reports and reminded participants before the interview of the possibility of a monitor placement request.

Our primary outcome was self-reported, full home smoking bans at both follow-ups. A full ban was defined as not allowing smoking anywhere inside the home, a partial ban allowed smoking in some places or at certain times or by some people and no ban if smoking was allowed anywhere, without rules. ${ }^{59}$

At baseline, we asked smokers the number of cigarettes smoked per day, quit attempts (daily smokers only) and confidence in quitting, ${ }^{60}$ as possible spillover effects not targeted directly by the programme.

Descriptors included participants' baseline demographic characteristics (gender, race/ethnicity, employment, income, education, marital/partner status and age), smoking status and number of cigarettes smoked per day and household composition. At both follow-ups, participants reporting partial or full bans were asked about enforcement problems, 'How often are your smoking rules broken by someone?' (never/rarely/sometimes/very often).

The intervention process was measured by three sources. The tracking tool captured coaching calls completed and returned mailings. During the coaching call, respondents were asked whether they received mailing \#1. The 3-month interview included questions about the receipt of materials, the extent they were read (none/some/most/all) and their personal relevance and usefulness and about the receipt of the coaching call and its usefulness and satisfaction (not at all/a little/somewhat/very).

\section{Statistical analyses}

Our analyses were specified in advance and replicated those from the previous trials. We calculated descriptive statistics of all key variables and then examined univariate and bivariate distributions for all relevant variables for each time point. In addition, we investigated patterns of missing data and group differences due to randomisation for all baseline demographic, smoking and household composition variables. Subsequently, we used hierarchical linear growth modeling to assess the intervention impact on all primary and secondary outcomes (intent-to-treat) with binary logistic (full/partial or no banbinary outcome), ordinal logistic (full/partial/no ban), Poisson (quit attempts) and linear multilevel models (number of cigarettes/day, confidence in quitting). ${ }^{61}$ This allowed us to model all available data (intent-to-treat), including all participants with a minimum of baseline data, that is, all randomised participants. On the basis of preliminary results from investigating trajectories, we modelled linear change over time and modelled the cross-level interaction effect of time and group assignment to assess the effectiveness of the intervention. We also conducted more conservative sensitivity analyses using the same growth curve models. Sensitivity analysis \#1 assumed that all participants without follow-up data were failures. A second, very conservative sensitivity analysis added those who reported enforcement problems to those assumed to be failures. Analyses were conducted using SAS V.9.4 and HLM7.

\section{RESULTS}

\section{Participants}

Of 9953 callers asked whether there was a smoker living in the household, $8.8 \%$ refused or were not interested in the programme/study (figure 1 ). Of the remainder, $17.4 \%$ reported $\geq 1$ smoker; 498 (31.5\%) of those with $\geq 1$ smoker declined further screening. Of the 625 who met all criteria, $81.3 \%$ enrolled. The most important reasons for loss were no household smoker and no interest in the programme/study.

Participants were predominantly women $(82.9 \%)$, living in a single-smoker household (76.4\%), not employed (71.3\%), a smoker $(71.3 \%)$, living with one or more children $<18$ years (68.3\%), African-American (65.2\%) and single, not living with a partner $(58.3 \%$ ) (table 2 ). Half were very low income, $\leq$ US\$10 000/year (49.6\%). At baseline, the majority $(59.3 \%)$ reported having a partial ban, and the remainder had no home smoking restrictions. Study groups differed significantly on gender $(p=0.003)$ and race/ethnicity $(p=0.03)$, with the intervention group having larger proportions of women and African-Americans (table 2).

Follow-up was completed at 3 months by $72.2 \%$ of enrollees and at 6 months by $66.1 \%$ (figure 1). There were two missing data patterns at both follow-ups. More African-Americans $(76.4 \%)$ were reached at 3 months than whites $(62.5 \%$; $\mathrm{p}<0.01)$ and at 6 months $(70.1 \%$ vs $51.0 \% ; \mathrm{p}<0.001)$, and fewer intervention participants $(67.4 \%)$ than controls $(77.2 \%)$ were followed at 3 months $(p=0.01)$. Similarly, fewer intervention participants $(61.6 \%)$ than controls $(70.8 \%)$ completed 6-month interviews $(\mathrm{p}=0.03)$.

\section{Intervention delivery and response}

According to the tracking tool, 253 (98\%) of the 258 intervention participants did not have any mailing returned, and $186(72.1 \%)$ completed a coaching call. All coaching call recipients said that they had received mailing \#1 before the call. At the 3 -month interview, $92.5 \%$ of interviewees reported receiving all of the materials; $72.1 \%$ said that they had received the coaching call. Most interviewees (74.4\%) reported reading most/all materials, rating them as follows: related to them personally, very $=48.8 \%$, somewhat $=34.4 \%$; useful, very $=58.8 \%$, somewhat $=30.0 \%$. The coaching call was rated as follows: related to them personally, very $=48.4 \%$, somewhat $=31.2 \%$; useful, very $=70.5 \%$, somewhat $=18.0 \%$; satisfied, very $=85.3 \%$, somewhat $=9.0 \%$.

At 3 months, intervention participants reported whether they had taken programme-recommended intermediate actions: $73.1 \%$ talked with family about a ban, $66.9 \%$ made a list of reasons for a smoke-free home, $65.6 \%$ used the provided stickers and $54.4 \%$ put up the signs. Some $(11.9 \%)$ reported that they or someone in their household called a smoking cessation service. 


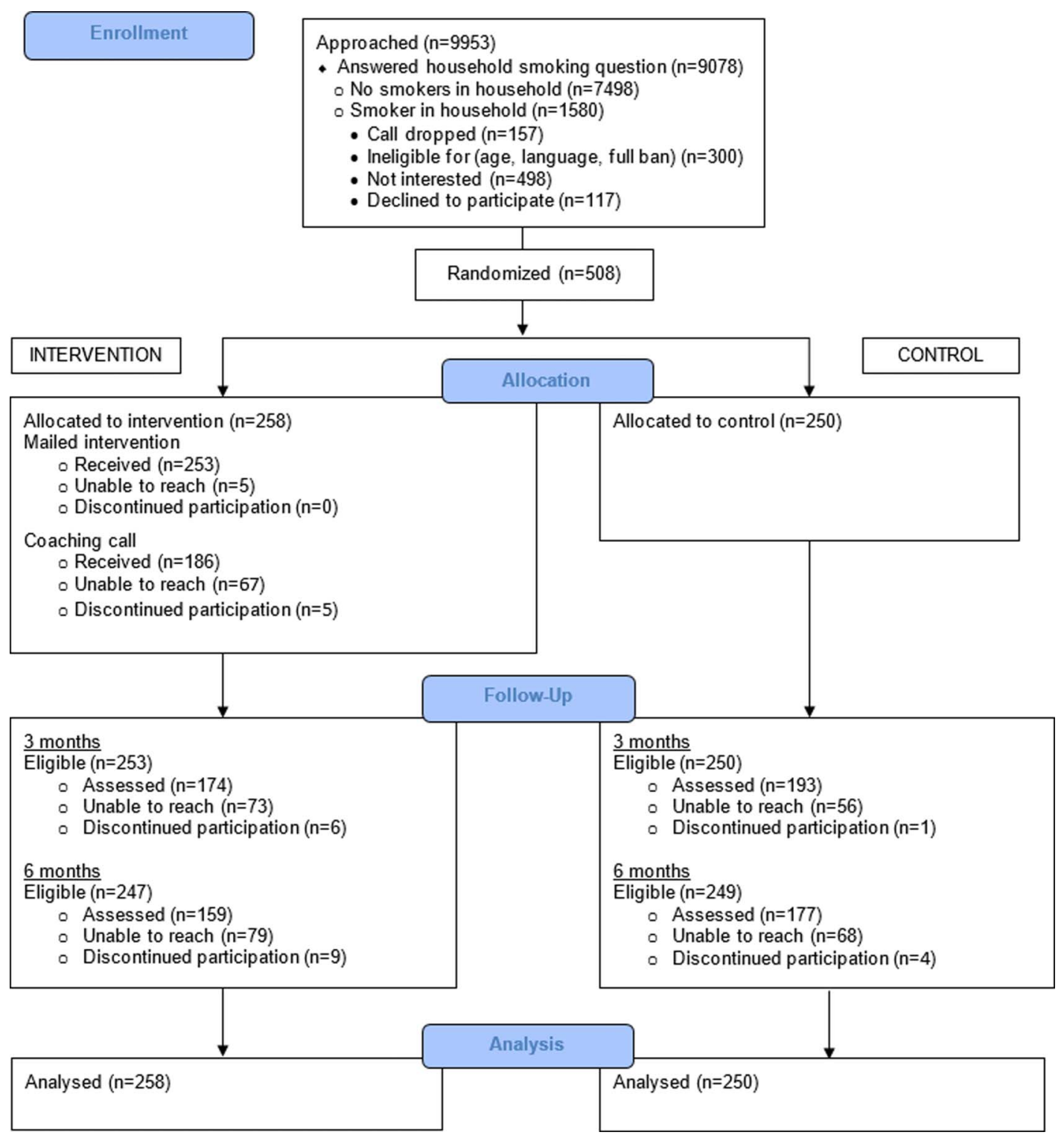

Figure 1 Consolidated Standards of Reporting Trials (CONSORT) flow diagram.

\section{Intervention impact on primary and secondary outcomes Primary outcome}

Intervention participants were more likely than controls to report a full ban at both follow-ups. At 3 months, the proportions were $46.6 \%$ and $25.4 \%(\mathrm{p}<0.0001)$ and at 6 months, they were $62.9 \%$ and $38.4 \%(\mathrm{p}<0.0001)$, respectively (table 3$)$. The longitudinal intent-to-treat analysis showed a significant intervention effect over time. At 3 months, the model OR was 1.48 (95\% CI 1.241 to $1.772, \mathrm{p}=<0.0001)$. This results in a predicted OR of 2.19 at 6 months, that is, 1.48 squared at the follow-up 2. ${ }^{62}$ Treating the ban as an ordinal variable (full/ partial/no ban) also resulted in a significant treatment effect (table 3).

Exploratory results for Latinos $(n=61$ at baseline, $n=45$ at 3 months follow-up, $n=42$ at 6 months follow-up) indicated that $52.2 \%$ of the intervention group reported a full ban at 3 months compared with $36.4 \%$ of controls $(p=0.29)$, and at 6 months, $57.1 \%$ of both groups reported a full ban $(p=1.00)$.

Sensitivity analyses maintained statistically significant differences favouring the intervention group, although not in the growth model for the second very conservative sensitivity analysis. When those lost to follow-up were defined as failures, results were $31.4 \%$ vs $19.6 \%(p=0.002)$ at 3 months and $38.8 \%$ vs $27.2 \% \quad(\mathrm{p}=0.006)$ at 6 months (growth model $\mathrm{OR}=1.23$, 95\% CI (1.073 to 1.409$), \mathrm{p}=0.003)$. When we defined success as only those reporting a full ban and no enforcement problems, the pattern was similar, with $17.1 \%$ vs $8.8 \%(\mathrm{p}=.006)$ at 3 months and $24.0 \%$ vs $16.0 \%(\mathrm{p}=0.02)$ at 6 months (growth model OR $=1.13,95 \%$ CI $(0.951$ to 1.342 ), $\mathrm{p}=0.164)$.

\section{Secondary outcomes}

Baseline smokers reported fewer cigarettes smoked daily (at both 3 months and in the intent-to-treat (ITT) analysis; however, their numbers of quit attempts and confidence in quitting did not differ (table 3).

\section{Comparison of the three trials}

Our participants differed from those in the other trials in that they had fewer smokers in the household, and they reflected the greater racial and ethnic diversity of the Texas Gulf Coast region. Our trial included more Latinos (12.0\%) than Atlanta 
Table 2 Study participants' baseline demographic, smoking and household characteristics

\begin{tabular}{|c|c|c|c|c|}
\hline Characteristics & $\begin{array}{l}\text { Total }(n=508), \text { no. }(\%) \text { or } \\
\text { mean } \pm \text { SD }\end{array}$ & $\begin{array}{l}\text { Intervention }(n=258), \text { no. }(\%) \text { or } \\
\text { mean } \pm \text { SD }\end{array}$ & $\begin{array}{l}\text { Control }(n=250), \text { no. }(\%) \text { or } \\
\text { mean } \pm \text { SD }\end{array}$ & p Value \\
\hline Female & $421(82.9)$ & $223(86.4)$ & $198(79.2)$ & 0.003 \\
\hline \multicolumn{5}{|l|}{ Race/ethnicity } \\
\hline African-American & $331(65.2)$ & $180(69.8)$ & $151(60.4)$ & \\
\hline Latino & $61(12.0)$ & $33(12.8)$ & $28(11.2)$ & \\
\hline White & $96(18.9)$ & $40(15.5)$ & $56(22.4)$ & \\
\hline Other & $20(3.9)$ & $5(1.9)$ & $15(6.0)$ & 0.03 \\
\hline \multicolumn{5}{|l|}{ Employment } \\
\hline Employed & $146(28.7)$ & $69(26.7)$ & 77 (30.8) & \\
\hline Unemployed/homemaker/retired/disabled/other & $362(71.3)$ & $189(73.3)$ & $173(69.2)$ & 0.31 \\
\hline \multicolumn{5}{|l|}{ Income } \\
\hline$\leq$ US\$10 000 & $252(49.6)$ & $124(48.1)$ & $128(51.2)$ & \\
\hline US\$10 001-US\$20000 & $152(29.9)$ & $82(31.8)$ & $70(28.0)$ & \\
\hline US\$20 001-US\$35000 & $65(12.8)$ & $32(12.4)$ & $33(13.2)$ & \\
\hline US\$35 001-US\$50 000 & $19(3.7)$ & $11(4.3)$ & $8(3.2)$ & \\
\hline$\geq$ US\$50 001 & $14(2.8)$ & $7(2.7)$ & $7(2.8)$ & 0.52 \\
\hline Missing & $6(1.2)$ & $2(0.7)$ & $4(1.6)$ & \\
\hline \multicolumn{5}{|l|}{ Education } \\
\hline Less than/some high school & $109(21.5)$ & $54(20.9)$ & $55(22.0)$ & \\
\hline High school graduate/General education certificate & $213(41.9)$ & $111(43.0)$ & $102(40.8)$ & \\
\hline Vocational/technical school/some College & $161(31.7)$ & $82(31.8)$ & 79 (31.6) & \\
\hline College graduate or higher & $25(4.9)$ & $11(4.3)$ & $14(5.6)$ & 0.89 \\
\hline \multicolumn{5}{|l|}{ Marital status } \\
\hline Not married, living with partner & $114(22.4)$ & $63(24.4)$ & $51(20.4)$ & \\
\hline Married & $97(19.1)$ & $43(16.7)$ & $54(21.6)$ & \\
\hline Single & $296(58.3)$ & $152(58.9)$ & $144(57.6)$ & 0.28 \\
\hline Missing & $1(0.2)$ & - & $1(0.4)$ & \\
\hline Age in years & $41.1 \pm 12.65$ & $40.4 \pm 12.42$ & $41.9 \pm 12.86$ & 0.16 \\
\hline Smoker & $362(71.3)$ & $183(70.9)$ & $179(71.6)$ & 0.87 \\
\hline Number of cigarettes per day* & $11.7 \pm 8.87$ & $10.9 \pm 8.84$ & $12.5 \pm 8.84$ & 0.08 \\
\hline \multicolumn{5}{|l|}{ Number of smokers in the home } \\
\hline 1 & $388(76.4)$ & 194(75.2) & $194(77.6)$ & \\
\hline 2 & $56(11.0)$ & $32(12.4)$ & $24(9.6)$ & \\
\hline$\geq 3$ & $64(12.6)$ & $32(12.4)$ & $32(12.8)$ & 0.53 \\
\hline \multicolumn{5}{|l|}{ Number of non-smoking adults in the home } \\
\hline 0 & $129(25.4)$ & $66(25.6)$ & $63(25.2)$ & \\
\hline 1 & $275(54.1)$ & $134(51.9)$ & $141(56.4)$ & \\
\hline$\geq 2$ & $104(20.5)$ & $58(22.5)$ & $46(18.4)$ & 0.55 \\
\hline \multicolumn{5}{|l|}{ Age of children in the home (years) } \\
\hline $5-18$ & $181(35.7)$ & $92(35.6)$ & 89 (35.6) & 0.27 \\
\hline $1-4$ & $116(22.8)$ & $63(24.4)$ & $53(21.2)$ & 0.28 \\
\hline$<1$ & $50(9.8)$ & $27(10.5)$ & $23(9.2)$ & 0.63 \\
\hline No children $<18$ & $161(31.7)$ & 76 (29.5) & $85(34.0)$ & \\
\hline \multicolumn{5}{|l|}{ Home smoking ban status } \\
\hline Partial ban & $301(59.3)$ & $148(57.4)$ & $153(61.2)$ & \\
\hline No ban & $207(40.7)$ & $110(42.6)$ & $97(38.8)$ & 0.38 \\
\hline
\end{tabular}

$(1.8 \%)$ or NC $(3.0 \%)(\mathrm{p}<0.0001)$ and fewer African-Americans $(p<0.0001)$ for both comparisons. Furthermore, because the programme targeted English speakers, we missed opportunities to reach still more Latinos. At baseline, fewer Texas participants


$\mathrm{p}=0.007)$, and fewer had children under 18 years in the household compared with the other sites $(p<0.0001$ for both comparisons); many more Texas participants lived in households with a single smoker (76.4\%) than Atlanta $(50.0 \%)$ or $\mathrm{NC}$ (48.0\%) ( $p<0.0001$ for both comparisons). Texas participants were similar in median age and proportions of participants who were women, single, had high school/GED or less education, very low income and partial or no bans at baseline (all comparisons $=\mathrm{p}>0.05$, data not shown).

The Texas Gulf Coast, NC and Atlanta 2-1-1 call centres all offer 24/7/365 coverage and services in multiple languages, although the Texas 2-1-1 call centre volume of 2500 calls/day is substantially larger than the other two (table 1). As noted in the 'Introduction' section, smoking prevalence in the Gulf Coast (based on 2010 BRFSS data) is 1-2 percentage points lower than Atlanta and 5-6 points lower than NC (table 1). 


\begin{tabular}{|c|c|c|c|c|c|c|c|c|}
\hline & \multicolumn{3}{|c|}{ 3-month assessment } & \multicolumn{3}{|c|}{ 6-month assessment } & \multicolumn{2}{|c|}{$\begin{array}{l}\text { Intent-to-treat } \\
\text { analysis } \\
\text { intervention group } \\
\text { change* }^{*}\end{array}$} \\
\hline & $\begin{array}{l}\text { Intervention } \\
(\mathrm{n}=174), \text { no. }(\%) \\
\text { or mean } \pm \text { SD }\end{array}$ & $\begin{array}{l}\text { Control } \\
(n=193), \text { no. }(\%) \\
\text { or mean } \pm \text { SD }\end{array}$ & $\mathrm{p}$ Value & $\begin{array}{l}\text { Intervention } \\
(n=159), \text { no. }(\%) \\
\text { or mean } \pm \text { SD }\end{array}$ & $\begin{array}{l}\text { Control } \\
(n=177), \text { no. }(\%) \\
\text { or mean } \pm \text { SD }\end{array}$ & $\mathrm{p}$ Value & Effect $\dagger$ & p Value \\
\hline \multicolumn{9}{|l|}{ Primary outcome } \\
\hline \multicolumn{9}{|l|}{ Home smoking ban } \\
\hline Full ban & $81(46.6)$ & $49(25.4)$ & $<0.0001 \ddagger$ & $100(62.9)$ & $68(38.4)$ & $<0.0001 \ddagger$ & $1.48 \ddagger$ & $<0.0001 \ddagger$ \\
\hline No full ban & $93(53.4)$ & $144(74.6)$ & & $59(37.1)$ & $109(61.6)$ & & & \\
\hline Partial ban & $58(33.3)$ & $89(46.1)$ & $0.0001 \S$ & $39(24.5)$ & $59(33.3)$ & $<0.0001 \S$ & $1.61 \S$ & $<0.001 \S$ \\
\hline No ban & $35(20.1)$ & $55(28.5)$ & & $20(12.6)$ & $50(28.3)$ & & & \\
\hline \multicolumn{9}{|c|}{ Secondary outcomes_-baseline smokers only } \\
\hline Smokers & $(n=113)$ & $(n=127)$ & & $(n=96)$ & $(n=107)$ & & & \\
\hline No. of cigs/day & $7.6 \pm 8.1$ & $10.0 \pm 8.2$ & 0.03 & $7.5 \pm 8.1$ & $9.1 \pm 6.8$ & 0.13 & 0.30 & 0.04 \\
\hline Quit attempts past 3 months & $1.6 \pm 1.9$ & $1.3 \pm 1.4$ & 0.20 & $1.1 \pm 1.4$ & $1.0 \pm 1.5$ & 0.65 & 0.42 & 0.78 \\
\hline Confidence in quitting & $7.0 \pm 2.8$ & $6.5 \pm 2.5$ & 0.16 & $7.0 \pm 2.9$ & $6.4 \pm 2.4$ & 0.09 & 0.11 & 0.08 \\
\hline
\end{tabular}

Compared with findings from Atlanta and NC (6-month ITT ORs $=1.56$ and 1.72, respectively), the Texas OR of 2.19 indicated greater success at creating full bans. Two considerations, however, lead us to conclude that the trial results are comparable. First, Atlanta results were likely influenced by the threat of being asked to place a nicotine monitor in the home, that is, a 'bogus pipeline' effect. ${ }^{63}$ (Comparison of the monitor results and self-report in Atlanta did not differ statistically, but neither the NC nor Texas trials used monitors and, thus, did not convey the 'threat' of verification.) Second, compared with NC, the predominance of single-smoker households and fewer non-Latino white smokers in Texas likely favoured a larger effect.

We approached more than twice as many callers as did Atlanta or NC to reach the enrolment target. Fewer of Texas Gulf Coast callers reported a smoker, and among those callers, more (by almost twice) said that they were not interested and discontinued screening (table 4). We explored the possibility that the Houston Housing Authority smoke-free ordinance affected willingness to respond to the household smoker question, but few who refused (1.34\%) resided in public housing.

\section{DISCUSSION}

This successful replication provides clear external validity of the Atlanta trial as well as the NC trial, a result rarely attempted or achieved. The three trials demonstrate that, in the context of subtle systematic differences in methods, and subtle difference in settings and populations/samples, and differences in time, we could still attain essentially the same results. Five dissemination field tests currently in progress will provide the capstone evidence of feasibility when recruitment and counselling services are provided by independent service delivery 211 teams across the USA.

The programme's low intensity is unique compared to virtually all prior interventions that have featured multiple or more intensive counselling sessions and is unusual in including households without children. ${ }^{18} \quad 19$ 23-27 6465 Our goal of reduced exposure rather than cessation alone is, however, aligned with most previously tested interventions. ${ }^{19} 23-276465$
Failure to detect comparable impact of evidence-based interventions delivered in new settings can often be traced to problems with inadequate implementation, fidelity and level of implementation. ${ }^{54}$ Further, most interventions are adapted in some way during implementation in new settings/populations. This was not the case in the Texas and NC replications, which maintained strong initial and ongoing implementation support for this relatively simple programme. Delivery of the intervention materials by mail in all three trials was nearly $100 \%$, assisted by the address checker in the tracking tool. On the other hand, Texas delivered fewer coaching calls (72\% vs $92 \%$ and $81 \%$ in Atlanta and NC, respectively), in part because an estimated $30 \%$ of telephone numbers were out of service only 2 weeks after the baseline interview.

Table 4 Three-site comparison of eligibility and consent rates, coaching call delivery and follow-up

\begin{tabular}{|c|c|c|c|}
\hline & $\begin{array}{l}\text { Texas } \\
\text { No. (\%) }\end{array}$ & $\begin{array}{l}\text { NC } \\
\text { No. }(\%)\end{array}$ & $\begin{array}{l}\text { Atlanta } \\
\text { No. (\%) }\end{array}$ \\
\hline Approached & 9953 & 3422 & - \\
\hline $\begin{array}{l}\text { Answered question re: smoker in } \\
\text { the household }\end{array}$ & $9078(91.2)^{*}$ & $3236(94.6)$ & 4175 \\
\hline$\geq 1$ smoker in household & $1580(17.4) \dagger$ & 949 (29.3) & $1202(28.8)$ \\
\hline $\begin{array}{l}\text { Not interested in the programme/ } \\
\text { study }\end{array}$ & 498 (31.5)‡ & 149 (15.7) & 212 (17.6) \\
\hline Call dropped & 157 (9.9)‡ & $20(2.1)$ & $17(1.4)$ \\
\hline Ineligible-other & $300(19.0) \ddagger$ & $188(19.8)$ & $351(29.2)$ \\
\hline Met all eligibility criteria & 625 (39.6)‡ & $592(62.4)$ & $622(51.7)$ \\
\hline Consented and enrolled & $508(81.2) \S$ & $500(84.5)$ & $498(80.1)$ \\
\hline Coaching call & 186 (72.1)\# & $211(81.2)$ & $227(92.3)$ \\
\hline 3-month follow-up & 367 (72.2)\# & 412 (82.4) & $414(83.1)$ \\
\hline 6-month follow-up & 336 (66.1)\# & $380(76.0)$ & $394(79.1)$ \\
\hline \multicolumn{4}{|c|}{$\begin{array}{l}\text { *Number of approached. } \\
\text { †Number of participants who answered question re: smoker in the household. } \\
\text { ¥Number of } \geq 1 \text { smoker in the household. } \\
\text { \$Number of participants who met all criteria. } \\
\text { \#Number of consented and enrolled. }\end{array}$} \\
\hline
\end{tabular}




\section{Lessons about recruitment efficiency and reach}

The most effective implementation and sustainability strategies are ones that can be easily modified to adapt to new contexts and changing environments. This view is consistent with the perspective of continuous quality improvement and recognises that to enhance sustainability, implementation approaches should be flexible. ${ }^{54}$ Our trial suggests the need for adaptive changes in recruitment. Further work on the timing and wording of the household smoking question might increase response rates and disclosure, for example, creating a multiple choice question rather than asking a simple 'yes'/'no' question. ${ }^{66}{ }^{67}$ Disclosure may be especially important, although our data did not permit us to say how much low reports of household smokers resulted from regional smoking prevalence versus reluctance to disclose.

To improve efficiency in areas with lower smoking prevalence, we suggest combining screening and recruitment for this programme with other programmes, building on the portfolio of specialised programmes common among 2-1-1 systems, for example, among veterans. ${ }^{30}$ Further, new recruitment options are presented as more clients access 2-1-1 services through the internet. It is not clear whether callers who expressed disinterest were reacting to the research study in general or to this programme in particular and whether the programme was perceived as requiring cessation. Assurance during recruitment that the goal is not cessation might reduce resistance. Information about monetary incentives will be available from the dissemination field tests.

\section{Extension to new populations}

Exploratory results for English-speaking/bilingual Latinos suggest that the programme had an initial effect in this more highly acculturated Latino group. A possible measurement effect in the control group also merits consideration. Adaptation of materials and messages to address different barriers, contextual issues and cultural considerations would potentially strengthen the effect, together with translation for Spanish-speakers and bilingual, multigenerational households. ${ }^{68}$ Adaptation for American Indians/Alaska Native families is underway (Kegler MC, 4 February 2016), and an adapted version of the intervention for use in China showed promising results. ${ }^{69}$

\section{CONCLUSIONS}

Programmes that target home SHSe may serve as a 'Trojan horse' to change tobacco culture. ${ }^{25}$ Following the demonstrations of utility in these three trials, it may be possible to link such programmes to advances in public policies that support them and to add formal regulatory practices, as now proposed, banning smoking in federally subsidised housing. ${ }^{70}$ Future analyses should be directed towards assessing these complex and interacting contingencies.

The robust effect demonstrated in our trial, even with conservative sensitivity analyses, illustrates the importance of programme design and testing, beginning with the strategic choice of provider, with the staffing and structure needed for programme delivery and with wide reach to the intended beneficiaries. This multisite series also meets the suggested criterion of three replications ${ }^{51}$ and underscores the careful step-down of trial elements and monitoring that will be tested further in the dissemination phase. In contrast to single-shot, highly controlled efficacy trials, this initiative provides essential elements for attaining a sustainable public health impact.

\section{What this paper adds}

- A unique, minimal intervention to promote home smoking bans in low-income households was successful in efficacy (Atlanta) and effectiveness (North Carolina) trials. Replication of interventions is reported infrequently, despite concerns about the failure to replicate findings from individual trials.

- Full implementation throughout a national delivery system with broad reach to households without bans requires further testing to provide robust evidence of effectiveness.

- This trial (Texas) was in a more diverse population and different smoking context, substantiated the programme's effectiveness and generalisability and identified areas for adaptive changes to increase reach.

Acknowledgements The authors thank 2-1-1 Texas United Way of Greater Houston for their continued efforts to make this study a success. They specifically thank David Smith, Vallery Arnold, Amy Ricalday, Gema Hernandez, Frances Potakey, Teresa Barragan, Debbie Washington, Tyreece Ceaser, John Danny Davidson and Lena Estrada. They also thank University of Texas School of Public Health graduate assistants Sara Fares, Victoria Gbenjo and Amy Wieczorek for conducting follow-up interviews. The authors are grateful to Dr Paul R. Swank for advice on data reporting and to four anonymous reviewers for their valuable comments.

Contributors PDM directed the trial as the principal investigator, drafted the article and adjudicated input from coauthors. LSS assisted with trial direction as the coprincipal investigator, wrote parts of the article and made major edits. ŁTB assisted with the design of the study, codeveloped the intervention, assisted with quality control of intervention delivery and data collection and made edits on the full article. $\mathrm{RH}$ assisted with the study design, completed all data analysis and made major contributions in writing and editing the results. MH assisted with study design and development of measures and wrote parts of the introduction and discussion. MEF established the relationship with 2-1-1 Texas/United Way HELPLINE, wrote parts of the introduction and discussion and reviewed the full article. JAAM managed day-to-day study activities, including tracking and quality control of data collection and intervention delivery, and contributed to the draft of the article. RSW advised on study design and measures, managed the design of the web-based tracking tool and edited the full article. MWK assisted in conceptualising the study, established the 2-1-1 partnership nationally, assisted in the development of measures and reviewed the full article. DJ managed the 2-1-1 Texas/United Way HELPLINE, United Way of Greater Houston, Houston, TX partnership, made edits to the methods section and reviewed the final article. MCK is the principal investigator of the Smoke-Free Homes study, conceptualised and directed the overall study, assisted in developing measures, wrote parts of the introduction and made major edits on the full article.

Funding This publication was supported by the National Cancer Institute's State and Community Tobacco Control Research Initiative (grant number U01CA154282). This trial is registered with ClinicalTrials.gov number NCT02097914.

Disclaimer The content is solely the responsibility of the authors and does not necessarily represent the official views of the National Cancer Institute or the National Institutes of Health.

\section{Competing interests None declared.}

Ethics approval The University of Texas Health Science Center School of Public Health Institutional Review Board approved this protocol (\#13-0902).

Provenance and peer review Not commissioned; externally peer reviewed.

Open Access This is an Open Access article distributed in accordance with the Creative Commons Attribution Non Commercial (CC BY-NC 4.0) license, which permits others to distribute, remix, adapt, build upon this work non-commercially, and license their derivative works on different terms, provided the original work is properly cited and the use is non-commercial. See: http://creativecommons.org/ licenses/by-nc/4.0/

\section{REFERENCES}

1 Centers for Disease Control and Prevention (CDC). Vital signs: nonsmokers' exposure to secondhand smoke-United States, 1999-2008. MMWR Morb Mortal Wkly Rep 2010;59:1141-6. 
2 Homa DM, Neff LJ, King BA, et al. Vital signs: disparities in nonsmokers' exposure to secondhand smoke-United States, 1999-2012. MMWR Morb Mortal Wkly Rep 2015;64:103-8.

3 Mills AL, White MM, Pierce JP, et al. Home smoking bans among US households with children and smokers. Opportunities for intervention. Am J Prev Med 2011;41:559-65.

4 Norman GJ, Ribisl KM, Howard-Pitney B, et al. Smoking bans in the home and car: do those who really need them have them? Prev Med 1999;29:581-9.

5 Kegler MC, Malcoe LH. Smoking restrictions in the home and car among rural Native American and white families with young children. Prev Med 2002;35:334-42.

6 King BA, Dube SR, Homa DM. Smoke-free rules and secondhand smoke exposure in homes and vehicles among US adults, 2009-2010. Prev Chronic Dis 2013; 10:E79.

7 US Department of Health and Human Services. The health consequences of smoking -50 years of progress: a report of the surgeon general. Washington, DC: Office of the Surgeon General, 2014.

8 Oberg M, Jaakkola MS, Woodward A, et al. Worldwide burden of disease from exposure to second-hand smoke: a retrospective analysis of data from 192 countries. Lancet 2011;377:139-46.

9 Pirkle JL, Bernert JT, Caudill SP, et al. Trends in the exposure of nonsmokers in the US population to secondhand smoke: 1988-2002. Environ Health Perspect 2006;114:853-8.

10 Centers for Disease Control and Prevention (CDC). Disparities in secondhand smoke exposure-United States, 1988-1994 and 1999-2004. MMWR Morb Mortal Wkly Rep 2008:57:744-7.

11 International Agency for Research on Cancer. Evaluating the effectiveness of smoke-free policies. In: Handbooks of cancer prevention, tobacco control. Lyon, France: World Health Organization, 2009:9-58

12 Pizacani BA, Martin DP, Stark MJ, et al. Household smoking bans: which households have them and do they work? Prev Med 2003;36:99-107.

13 Hyland A, Higbee C, Travers MJ, et al. Smoke-free homes and smoking cessation and relapse in a longitudinal population of adults. Nicotine Tob Res 2009;11:614-18.

14 Messer $\mathrm{K}$, Mills AL, White MM, et al. The effect of smoke-free homes on smoking behavior in the US. Am J Prev Med 2008;35:210-6.

15 Forster JL, Widome R, Bernat DH. Policy interventions and surveillance as strategies to prevent tobacco use in adolescents and young adults. Am J Prev Med 2007;33(6 Suppl):S335-9.

16 Biener L, Nyman AL. Effect of workplace smoking policies on smoking cessation: results of a longitudinal study. J Occup Environ Med 1999:41:1121-7.

17 Pizacani BA, Martin DP, Stark MJ, et al. A prospective study of household smoking bans and subsequent cessation related behaviour: the role of stage of change. Tob Control 2004:13:23-8.

18 Hopkins DP, Briss PA, Ricard CJ, et al. Reviews of evidence regarding interventions to reduce tobacco use and exposure to environmental tobacco smoke. Am J Prev Med 2001;20(2 Suppl):16-66.

19 Hovell MF, Zakarian JM, Matt GE, et al. Effect of counselling mothers on their children's exposure to environmental tobacco smoke: randomised controlled trial. BMJ 2000;321:337-42

20 Hovell MF, Meltzer SB, Wahlgren DR, et al. Asthma management and environmental tobacco smoke exposure reduction in Latino children: a controlled trial. Pediatrics 2002;110:946-56.

21 Hovell MF, Zakarian JM, Matt GE, et al. Counseling to reduce children's secondhand smoke exposure and help parents quit smoking: a controlled trial. Nicotine Tob Res 2009;11:1383-94.

22 Gehrman CA, Hovell MF. Protecting children from environmental tobacco smoke (ETS) exposure: a critical review. Nicotine Tob Res 2003;5:289-301.

23 Rosen LJ, Noach MB, Winickoff JP, et al. Parental smoking cessation to protect young children: a systematic review and meta-analysis. Pediatrics 2012;129:141-52

24 Tyc VL, Huang Q, Nicholson J, et al. Intervention to reduce secondhand smoke exposure among children with cancer: a controlled trial. Psychooncology 2013:22:1104-11.

25 Hovell MF, Hughes SC. The behavioral ecology of secondhand smoke exposure: pathway to complete tobacco control. Nicotine Tob Res 2009;11:1254-64.

26 Stotts AL, Green C, Northrup TF, et al. Feasibility and efficacy of an intervention to reduce secondhand smoke exposure among infants discharged from a neonatal intensive care unit. J Perinatol 2013;33:811-6.

27 Rosen L, Myers V, Winickoff JP, et al. Effectiveness of interventions to reduce tobacco smoke pollution in homes: a systematic review and meta-analysis. Int J Environ Res Public Health 2015;12:16043-59.

28 United Way Worldwide and Alliance for Information and Referral Systems. http:/l www.211us.org/index.htm (accessed 14 Jan 2016).

29 Purnell JQ, Kreuter MW, Eddens KS, et al. Cancer control needs of 2-1-1 callers in Missouri, North Carolina, Texas, and Washington. J Health Care Poor Underserved 2012;23:752-67.
30 Kreuter MW. Reach, effectiveness, and connections: the case for partnering with 2-1-1 to eliminate health disparities. Am J Prev Med 2012:43(Suppl 5):S420-1.

31 Kreuter MW, Eddens KS, Alcaraz Kl, et al. Use of cancer control referrals by 2-1-1 callers: a randomized trial. Am J Prev Med 2012;43(Suppl 5):S425-34.

32 Kegler MC, Bundy L, Haardorfer R, et al. A minimal intervention to promote smoke-free homes among 2-1-1 callers: a randomized controlled trial. Am J Public Health 2015;105:530-7.

33 Escoffery C, Kegler MC, Butler S. Formative research on creating smoke-free homes in rural communities. Health Educ Res 2009;24:76-86

34 Kegler MC, Escoffery C, Groff A, et al. A qualitative study of how families decide to adopt household smoking restrictions. Fam Community Health 2007;30:328-41.

35 Kegler MC, Escoffery C, Bundy L, et al. Pilot study results from a brief intervention to create smoke-free homes. J Environ Public Health 2012;2012:951426.

36 Kok G, Gottlieb NH, Peters GY, et al. A taxonomy of behaviour change methods: an Intervention Mapping approach. Health Psychol Rev 2015;10:297-312.

37 Bartholomew Eldredge L, Markham C, Ruiter R, et al. Planning health promotion programs: an intervention mapping approach. San Francisco, CA: Jossey-Bass, 2016.

38 Bandura A. Social foundations of thought and action a social cognitive theory. Englewood Cliffs, NJ: Prentice-Hall, 1986.

39 Prochaska JO, DiClemente CC. Transtheoretical therapy: toward a more integrative model of change. Psychotherapy 1982;19:276-88.

40 Glasgow RE. What does it mean to be pragmatic? Pragmatic methods, measures, and models to facilitate research translation. Health Educ Behav 2013;40:257-65.

41 Factors Report for by County. http://statecancerprofiles.cancer.gov/risk/index.php? topic $=$ smoke $\&$ risk $=v 19 \&$ race $=00 \&$ sex $=0 \&$ datatype $=1 \&$ stateFIPS $=$

13\&type=risk\&sortVariableName=default\&sortOrder=default\#results (accessed 3 Mar 2016).

42 Centers for Disease Control and Prevention. Tobacco Control State Highlights 2012 http://www.cdc.gov/tobacco/data_statistics/state_data/state_highlights/2012/pdfs/ states/north_carolina.pdf (accessed 3 Mar 2016). State Cancer Profiles. Screening and Risk.

43 Williams RS, Stollings JH, Bundy $九$, et al. A Minimal Intervention to Promote SmokeFree Homes Among 2-1-1 Callers: North Carolina Randomized Effectiveness Trial. PLOS One 2016; In press.

44 Texas Department of Health and Human Services. Texas Behavioral Risk Factor Surveillance System Survey Data. 2010. https://www.dshs.state.tx.us/chs/brfss/query/ brfss form.shtm (accessed 7 Mar 2016).

45 Haines A, Kuruvilla S, Borchert M. Bridging the implementation gap between knowledge and action for health. Bull World Health Organ 2004;82:724-31.

46 Glasgow RE, Lichtenstein E, Marcus AC. Why don't we see more translation of health promotion research to practice? Rethinking the efficacy-to-effectiveness transition. Am J Public Health 2003:93:1261-7.

47 Glasgow RE, Green LW, Klesges LM, et al. External validity: we need to do more. Ann Behav Med 2006:31:105-8.

48 Green LW, Glasgow RE. Evaluating the relevance, generalization, and applicability of research: issues in external validation and translation methodology. Eval Health Prof 2006;29:126-53.

49 Camerer CF, Dreber A, Forsell $E$, et al. Evaluating replicability of laboratory experiments in economics. Science 2016;351:918

50 Gilbert DT, King G, Pettigrew $S$, et al. Comment on "Estimating the reproducibility of psychological science". Science 2016;351:1037.

51 Moonesinghe R, Khoury MJ, Janssens AC. Most published research findings are false-but a little replication goes a long way. PLoS Med 2007;4:e28

52 Mercer SL, DeVinney BJ, Fine $L$, et al. Study designs for effectiveness and translation research: identifying trade-offs. Am J Prev Med 2007:33:139-54.

53 Gartlehner G, Hansen RA, Nissman D, et al. Criteria for distinguishing effectiveness from efficacy trials in systematic reviews. Agency for Healthcare Research and Quality, 2006:1-19.

54 Chambers DA, Glasgow RE, Stange KC. The dynamic sustainability framework: addressing the paradox of sustainment amid ongoing change. Implement $\mathrm{SC}$ 2013;8:117.

55 SmartyStreets. 2016. https://smartystreets.com/ (accessed 28 Feb 2016).

56 Lairson DR, Huo J, Ricks KA, et al. The cost of implementing a 2-1-1 call center-based cancer control navigator program. Eval Program Plann 2013;39:51-6.

57 Hall KL, Stipelman BA, Eddens KS, et al. Advancing collaborative research with 2-1-1 to reduce health disparities: challenges, opportunities, and recommendations. Am J Prev Med 2012;43(Suppl 5):S518-28

58 Savas LS, Fernandez ME, Jobe $D$, et al. Human papillomavirus vaccine: 2-1-1 helplines and minority parent decision-making. Am J Prev Med 2012;43(Suppl 5): S490-6.

59 Centers for Disease Control and Prevention. 2008 Behavioral Risk Factor Surveillance System Questionnaire. 2007. http://www.cdc.gov/brfss/annual_data/ pdf-ques/2008brfss.pdf (accessed 15 Jan 2016)

60 Biener L, Abrams DB. The Contemplation Ladder: validation of a measure of readiness to consider smoking cessation. Health Psychol 1991;10:360-5.

61 Singer JD, Willett JB. Applied longitudinal data analysis modeling change and event occurrence. Oxford: Oxford University Press, 2003. 
62 Agresti A. An introduction to categorical data analysis. 2nd edn. Hoboken, NJ: Wiley-Interscience, 2007:104-5.

63 Jones EE, Sigall $H$. The bogus pipeline: a new paradigm for measuring affect and attitude. Psychol Bull 1971;76:349-64.

64 Winickoff JP, Hillis VJ, Palfrey JS, et al. A smoking cessation intervention for parents of children who are hospitalized for respiratory illness: the stop tobacco outreach program. Pediatrics 2003;111:140-5.

65 Emmons KM, Hammond SK, Fava JL, et al. A randomized trial to reduce passive smoke exposure in low-income households with young children. Pediatrics 2001;108:18-24.

66 Mullen PD, Carbonari JP, Tabak ER, et al. Improving disclosure of smoking by pregnant women. Am J Obstet Gynecol 1991;165:409-13.
67 Mullen PD, Carbonari JP, Glenday MC. Identifying pregnant women who drink alcoholic beverages. Am J Obstet Gynecol 1991;165(Pt 1):1429-30.

68 Savas LS, Mullen PD, Hovell, M., et al. Formative research for a Smoke-Free Homes (SFH) intervention for Hispanic Texan Households. Poster presentation, Innovations in Cancer Prevention and Research Conference; Austin, TX, 9-10 November 2015.

69 Wang J, Zhu S, Wong S, et al. Brief intervention to implement home bans on smoking: evaluating the findings from a randomised trial. Poster presentation. World Conference on Tobacco or Health. Abu Dhabi, United Arab Emirates, 17-21 March, 2015.

70 Regulations.gov. FR 5597-P-02 Instituting Smoke-Free Public Housing. 2016. http://www.regulations.gov/\#! IocketDetail;D=HUD-2015-0101 (accessed 7 Mar 2016). 